\title{
Effects of ornithine decarboxylase antizyme 1 on the proliferation and differentiation of human oral cancer cells
}

\author{
$\mathrm{XING}_{\mathrm{WANG}}{ }^{1,2}$ and $\mathrm{LI} \mathrm{JIANG}^{1}$ \\ ${ }^{1}$ Guangdong General Hospital, Guangdong Academy of Medical Sciences, Guangzhou, Guangdong 510080; \\ ${ }^{2}$ Shantou University Medical College, Shantou, Guangdong 515041, P.R. China
}

Received March 28, 2014; Accepted September 16, 2014

DOI: $10.3892 /$ ijmm.2014.1961

\begin{abstract}
Ornithine decarboxylase antizyme 1 (OAZ1) is an antizyme targeting ornithine decarboxylase for degradation, subsequently inhibiting polyamine production to prevent cell proliferation. OAZ1 is also involved in other major cellular events, including differentiation and apoptosis. Recent studies have shown that OAZ1 has tumor suppressor activities and its effects on cell proliferation and differentiation have been reported in several cancer cell lines. To explore the role of OAZ1 in human oral cancer, the effects of OAZ1 were studied on the proliferation and differentiation of human malignant oral cancer cell line, SCC15. MTT assay and flow cytometry analysis showed that stable $O A Z 1$ expression in SCC15 significantly inhibited cell proliferation $(\mathrm{P}<0.001)$ and induced $G_{0} / G_{1}$ arrest with the $G_{1}$-phase cells increased from 55.83 to $65.26 \%$. Morphological observation revealed the increased formation of epithelial islands. Further results from quantitative reverse transcription-polymerase chain reaction and western blot analysis proved the upregulation of several terminal differentiation marker genes $(K 10, F L G$ and $L O R$ ) in $O A Z 1$-expressed SCC15 cells. To elucidate the possible mechanism of $L O R$ upregulation by OAZ1, further experiments were performed and it was found that the $O A Z 1$ expression inhibited Smad nuclear interacting protein 1 (SNIP1) at the protein level and RNA interference of SNIP1 in SCC15 cells, which increased the expression of $L O R$. These results show that OAZ1 simultaneously inhibits the proliferation and induces the differentiation of oral cancer cells in humans. The effects on cellular differentiation depend partly on the degradation of SNIP1.
\end{abstract}

\section{Introduction}

Ornithine decarboxylase antizyme 1 (OAZ1) is a member of the ornithine decarboxylase (ODC) antizyme family, which

Correspondence to: Dr Li Jiang, Guangdong General Hospital, Guangdong Academy of Medical Sciences, 106 Zhongshan 2nd Road, Guangzhou, Guangdong 510080, P.R. China

E-mail: lijiang3434@yeah.net

Key words: ornithine decarboxylase antizyme 1, tongue squamous cancer cells, terminal differentiation targets ODC for ubiquitin-independent proteasome degradation, thereby inhibiting the synthesis of polyamines and cell proliferation (1). OAZ1 can also inhibit cell growth through ODC-independent mechanisms, including the inhibition of cellular uptake of polyamines by inactivating the polyamine uptake transporter, promoting cyclin D1 degradation and preventing ubiquitin-independent msp1 degradation (2-5). Studies of cancer have also proved that OAZ1 has tumor suppressor activities and it can affect the apoptosis and proliferation of multiple tumor cell lines (6-8).

Several investigators have studied the involvement of OAZ1 in tongue squamous cell carcinoma (TSCC). In p53-knockout mice, overexpression of the OAZ1 protein inhibited the production of keratinocyte proliferation marker, keratin $6(K 6)$, whereas it upregulated the expression of keratinocyte differentiation marker, loricrin (LOR) (9). In hamster malignant oral keratinocytes, ectopic expression of $O A Z 1$ induced epithelial differentiation with the overexpression of involucrin (IVL) (10). In several human oral cancer cell lines, the expression level of the $O A Z 1$ gene was downregulated $(11,12)$. A previous study has shown that OAZ1 induces the conversion of the human tongue squamous cancer cell line, UM1, to the less metastatic type, $\mathrm{UM} 2$, with the hypomethylation of genome DNA and histone $\mathrm{H} 3$ lysine 9 dimethylation (12). However, the overall effects of OAZ1 on cellular proliferation and differentiation in human oral cancer cells and the underlying mechanism remain to be studied.

In the present study, the lentiviral vector containing the $O A Z 1$ gene was constructed and transfected into the human tongue cancer cell line, SCC15, to evaluate the effects of $O A Z 1$ expression on proliferation and differentiation of the cells. The results showed that the stable expression of $O A Z 1$ in SCC15 inhibited the cell proliferation rate and induced $\mathrm{G}_{0} / \mathrm{G}_{1}$ arrest. $O A Z 1$ expression also induced the formation of epithelial islands with elevation of several differentiation marker genes $(K 10$, $F L G$ and $L O R$ ). OAZ1 was also found to inhibit Smad nuclear interacting protein 1 (SNIP1) and silencing of SNIP1 increased the expression of $L O R$ in SCC15 cells. The results of the study proved that OAZ1 can simultaneously inhibit proliferation and induce differentiation of oral cancer cells in humans.

\section{Materials and methods}

Cell culture and transfection. The human oral cancer cell line, SCC15, was obtained from the American Type Culture 
Table I. Primers used for RT-qPCR.

\begin{tabular}{|c|c|c|}
\hline Primer & Sequence $\left(5^{\prime} \rightarrow 3^{\prime}\right)$ & Product length (bp) \\
\hline OAZ1 (NM_004152) & $\begin{array}{l}\text { F: TCTCCCTCCACTGCTGTAGTAACC } \\
\text { R: GTTGAGAATCCTCGTCTTGTCGTT }\end{array}$ & 198 \\
\hline E-cadherin (NM_004360) & $\begin{array}{l}\text { F: CTACAATGCCGCCATCGCTTA } \\
\text { R: CACTGATGACTCCTGTGTTCCTGTT }\end{array}$ & 98 \\
\hline K1 (NM_006121) & $\begin{array}{l}\text { F: CGGAACTGAAGAACATGCAG } \\
\text { R: CATATAAGCACCATCCACATCC }\end{array}$ & 128 \\
\hline K6A(NM_005554) & $\begin{array}{l}\text { F: CAAGGCCCAATATGAGGAGA } \\
\text { R: GCAATCTCCTGCTTGGTGTT }\end{array}$ & 135 \\
\hline K10 (NM_000421) & $\begin{array}{l}\text { F: AAACCATCGATGACCTTAAAAATC } \\
\text { R: GCGCAGAGCTACCTCATTCT }\end{array}$ & 134 \\
\hline K19 (NM_002276) & $\begin{array}{l}\text { F: GCCACTACTACACGACCATCC } \\
\text { R: CAAACTTGGTTCGGAAGTCAT }\end{array}$ & 126 \\
\hline FLG (NM_002016) & $\begin{array}{l}\text { F: TTTCGGCAAATCCTGAAGAATCC } \\
\text { R: ACTGTGCTTTCTGTGCTTGTG }\end{array}$ & 195 \\
\hline IVL (NM_005547) & $\begin{array}{l}\text { F: CTGCCTCAGCCTTACTGTGA } \\
\text { R: TGGGTATTGACTGGAGGAGG }\end{array}$ & 133 \\
\hline LOR (NM_000427) & $\begin{array}{l}\text { F: GCACCGATGGGCTTAGAG } \\
\text { R: AGAAACCAAAGAGGCTAAACAG }\end{array}$ & 130 \\
\hline SNIP1 (NM_024700) & $\begin{array}{l}\text { F: AAGAAGCAAGTCTCCTCGCAG } \\
\text { R: GTTCTGATGGTTCCCTGTGCT }\end{array}$ & 128 \\
\hline GAPDH (NM_002046) & $\begin{array}{l}\text { F: CGCTGAGTACGTCGTGGAGTC } \\
\text { R: GCTGATGATCTTGAGGCTGTTGTC }\end{array}$ & 172 \\
\hline
\end{tabular}

Collection (ATCC, Manassas, VA, USA) and maintained in Dulbecco's modified Eagles medium/F12 medium supplemented with $10 \%(\mathrm{v} / \mathrm{v})$ fetal bovine serum at $37^{\circ} \mathrm{C}$ in $5 \% \mathrm{CO}_{2}$. The lentiviral vector (pLVX-Neo-IRES-ZsGreen) containing the green fluorescent protein (GFP) gene, ZsGreen, was ligated to $O A Z 1$ cDNA (loss of the frameshift site) to construct the stable expression vector of $O A Z 1$ (13). The cells were transferred to 6 -well plates and the $O A Z 1$-containing vector or control vector was transfected. When GFP was expressed, $\mathrm{G} 418(1,000 \mu \mathrm{g} / \mathrm{ml})$ and flow cytometry were used to screen the stably transfected clones. SCC15 cells transfected with the $O A Z 1$-expressed vector or the negative-control vector are referred as SCC15/OAZ1 and SCC15/GFP.

Cell proliferation assay. Cells (1×104/well) were transferred in 3 replicates to 96 -well plates and cultured on standard conditions. At the time-points of 24,48 and $72 \mathrm{~h}$, the viability of the cells was analyzed after the addition of $20 \mu \mathrm{l}$ MTT $(5 \mathrm{mg} / \mathrm{ml})$ and dimethyl sulfoxide. Absorbance was determined using the Multiskan MK3 Microplate Reader (Thermo Scientific, Waltham, MA, USA) at a wavelength of $490 \mathrm{~nm}$.

Cell cycle assay. Cells ( $2 \times 10^{5} /$ well) were transferred in 3 replicates to 6 -well plates. After $24 \mathrm{~h}$ of starvation, the cells were recovered for $36 \mathrm{~h}$ before analysis. The cells were treated according to the manufacturer's instructions for the cell cycle detection kit (Nanjing KeyGen Biotech, Nanjing, China) and submitted to flow cytometric analysis.

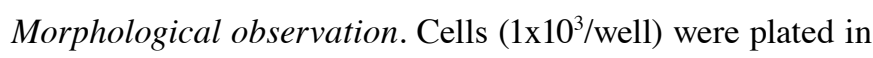
100x20 mm plates and observed under an inverted microscope after 1 week.

RNA interference. SNIP1-specific small hairpin RNA (shRNA) (GeneChem Co., Ltd., Shanghai, China) including sh-400 (5'-ATGCTGCTTTGAACAAGAC-3'), sh-401 (5'-TCGATGTATGTACATGACT-3'), sh-402 (5'-TTAGAC GTTCTCCTTTGGT-3') and the negative control, sh-NC (5'-TTCTCCGAACGTGTCACGT-3'), were synthesized. SCC15 cells were transferred to 6-well plates. When the cells reached $80 \%$ confluent, shRNA was transfected with Lipofectamine 2000 (Invitrogen, Carlsbad, CA, USA) in $250 \mu$ l Opti-MEM (Gibco, Carlsbad, CA, USA). Sh-NC was used as the negative control.

Reverse transcription-quantitative polymerase chain reaction (RT-qPCR) analysis. Total RNA was isolated using the RNAiso plus kit (Takara Bio, Inc., Shiga, Japan). Full-length cDNA was generated using the PrimeScript RT reagent kit (Takara, Dalian, China). cDNA was subjected to RT-qPCR analysis using SYBR-Green I (Takara) and results are reported as the ratio alteration among groups. The primers used are presented in Table I.

Immunoblotting. The cells were washed twice in phospate-buffered saline and lysed in radio-immunoprecipitation assay lysis buffer (with phenylmethanesulfonyl fluoride). Equal 
A

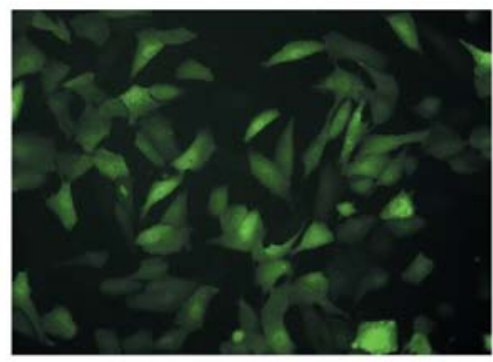

C

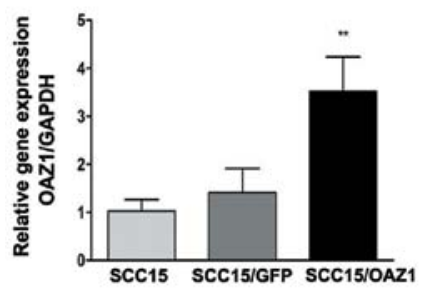

B

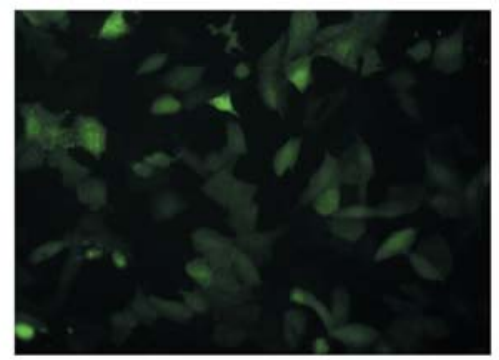

$\mathbf{E}$

Figure 1. Expression of ornithine decarboxylase antizyme 1 (OAZ1) in SCC15 cells. (A and B) Green fluorescent protein (GFP) expression detected by florescence microscope (magnification, x200) in SCC15 cells transfected with the (A) OAZ1-expressed vector or (B) control vector. (C-E) Confirmation of OAZ1 expression by (C) reverse transcription-quantitative polymerase chain reaction (RT-qPCR) and (D and E) western blotting. ${ }^{*} \mathrm{P}<0.05$ and ${ }^{* *} \mathrm{P}<0.01$ compared to the other groups.
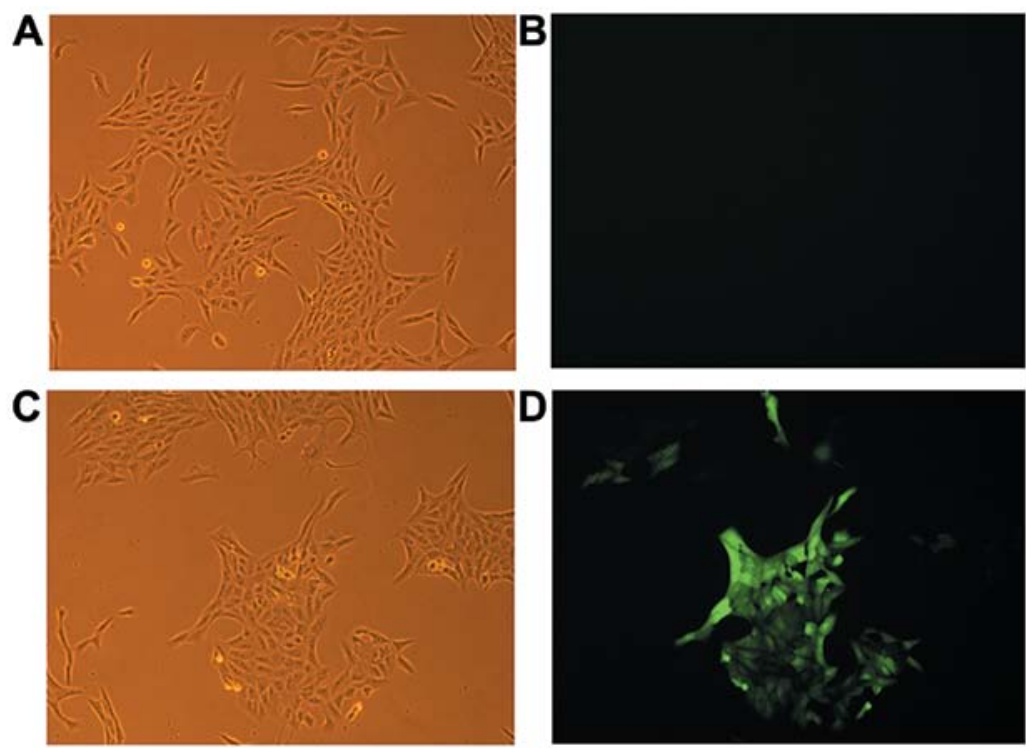

$\mathbf{E}$
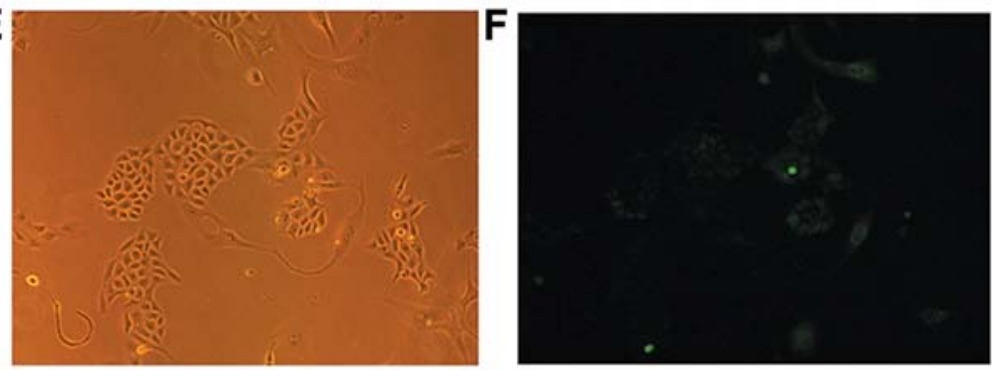

Figure 2. (A and B) Effect of ornithine decarboxylase antizyme 1 (OAZ1) overexpression on cell morphology in SCC15 cells (magnification, x100). SCC15 cells without treatment under a (A) regular or (B) fluorescence microscope. (C and D) SCC15 cells transfected with the control vector under a (C) regular or (D) florescence microscope. (E and F) SCC15 cells transfected with OAZ1-contained vector under a (E) regular or (F) florescence microscope.

amounts of total protein were separated by $12 \%$ SDS-PAGE, transferred to a polyvinylidene fluoride membrane (Millipore, Bedford, MA, USA) and probed with OAZ1 (Abcam, Hong Kong, China); K1, K10, IVL, LOR, filaggrin (FLG) and SNIP1 (Santa Cruz Biotechnology, Inc., Santa Cruz, CA, USA); and GAPDH (Cell Signaling Technology, Shanghai, China) antibodies and subsequently with the horseradish peroxidase-conjugated secondary antibody. The blots were evaluated with the ECL detection system (Advansta, Menlo Park, CA, USA). The protein bands of interest were quantified using 


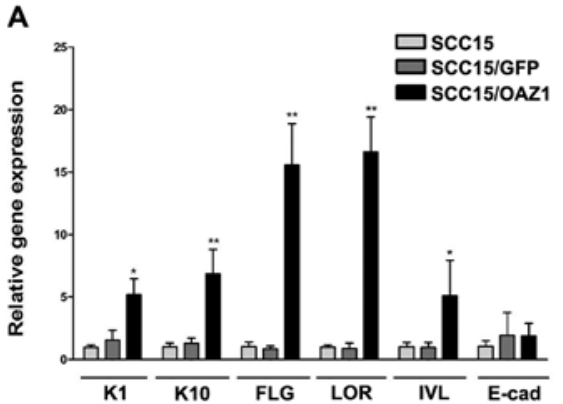

C

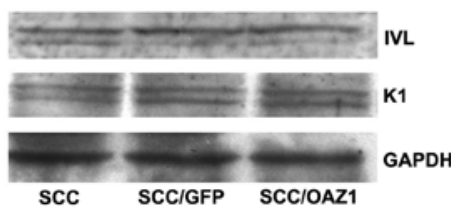

D

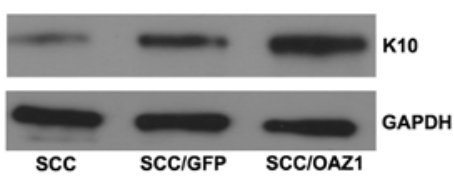

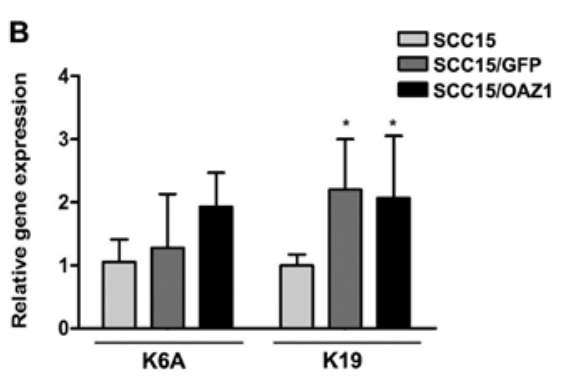

E

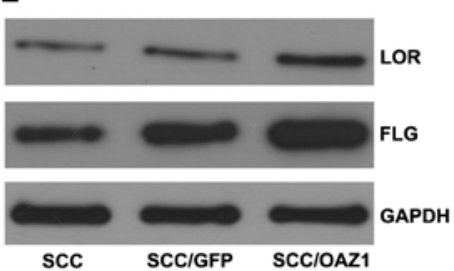

Figure 3. Effects of ornithine decarboxylase antizyme 1 (OAZ1) on differentiation and proliferation markers in SCC15 cells. (A and B) Reverse transcription-quantitative polymerase chain reaction (RT-qPCR) analysis of the (A) differentiation [keratin $1(K 1)$, K10, filaggrin $(F L G)$, involucrin $(I V L)$, loricrin $(L O R)$ and E-cadherin] and (B) proliferation markers (K6A and K19) among the different groups. (C-E) Western blotting detection of (C) K1, IVL, (D) K10, LOR and (E) FLG among the different groups. ${ }^{*} \mathrm{P}<0.05$ and ${ }^{* *} \mathrm{P}<0.01$ compared to the other groups.

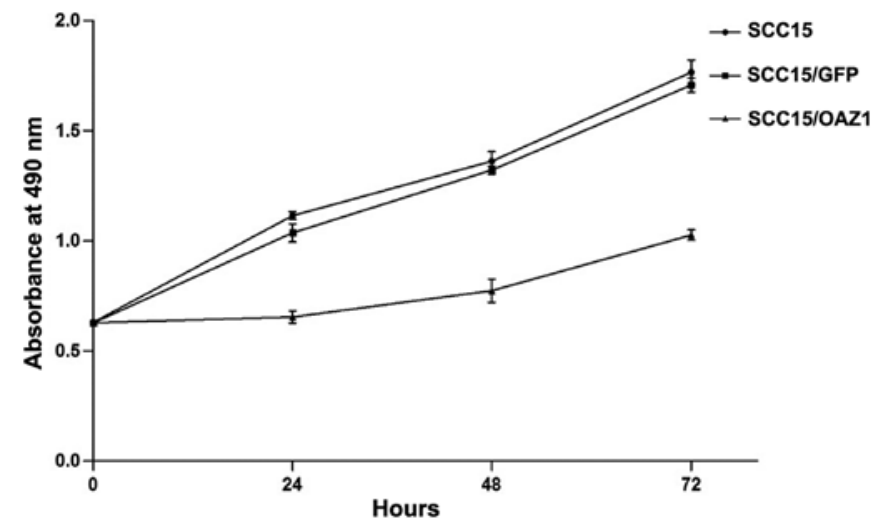

Figure 4. Ornithine decarboxylase antizyme 1 (OAZ1) inhibits cell proliferation in SCC15 cells. The curves show the viability of SCC15 cells detected by MTT analysis in the different groups.

FluorChem 8900 image analysis software (Alpha Innotech, San Leandro, CA, USA), and the integrated signal densities were normalized to GAPDH first, and subsequently expressed in terms of the fractional abundance relative to the control cells. These experiments were replicated 3 times.

Statistical analysis. SPSS 16.0 (SPSS, Inc., Chicago, IL, USA) was used for statistical analysis. Data are presented as means \pm standard deviation. One-way analysis of variance was used and $\mathrm{P}<0.05$ was considered to indicate a statistically significant difference.

\section{Results}

Overexpression of OAZ1 induces morphological changes in SCC15 cells. The lentiviral vector expressing $O A Z 1$ and GRF (ZsGreen), and the control vector expressing GFP were transfected into SCC15 cells separately and stable expression of GFP was observed in the two groups (Fig. 1A and B).
Quantitative analysis showed that the mRNA level of $O A Z 1$ was significantly elevated in the $O A Z 1$-transfected group (Fig. 1C). The OAZ1 protein expression was verified by western blotting (Fig. 1D). The cells transfected with the control vector (Fig. 2C and D) demonstrated the typical appearance of SCC15 (Fig. 2A and B). However, the appearance of the $O A Z 1$ transfectants exhibited evidence of epithelial island formation and increased cellular junctions indicating the terminal differentiation of the cells (Fig. 2E and F).

OAZ1 affects the expression of marker genes in cell differentiation. OAZ1-transfected SCC15 cells showed morphological alterations indicating terminal differentiation. To further confirm the effects of OAZ1 on the differentiation of SCC15, the expression of several reported differentiation marker genes were examined. Elevation of early terminal differentiation genes, $K 1, K 10$ and $I V L$, was observed in $O A Z 1$-expression cells. As for late terminal differentiation genes, the elevation of $F L G$ and $L O R$ was also observed, whereas the mRNA level of E-cadherin remained stable (Fig. 3A). When detected by western blot analysis, K10, LOR and FLG showed increased expression on protein level (Fig. 3C-E).

OAZ1 inhibits the proliferation of SCC15 cells. MTT assay was performed to detect the effects of $O A Z 1$ expression on the proliferation of SCC15. Compared to the control, SCC15 cells stably expressing $O A Z 1$ showed a significant reduction in the cell growth rate $(\mathrm{P}<0.001$ compared to the other groups) (Fig. 4), indicating that $O A Z I$ expression inhibited the proliferation of SCC15.

The cell cycle profiles of the OAZ1-transfected SCC15 cells and the control cells were further examined (Fig. 5). The flow cytometry analysis showed that the cells in the $\mathrm{G}_{1}$ phase were increased in $O A Z 1$-expressed SCC15 cells (65.26\%) compared to the control (55.83\%). Whereas cells in the $\mathrm{S}$ phase were decreased to $30.07 \%$ compared to the control 
A

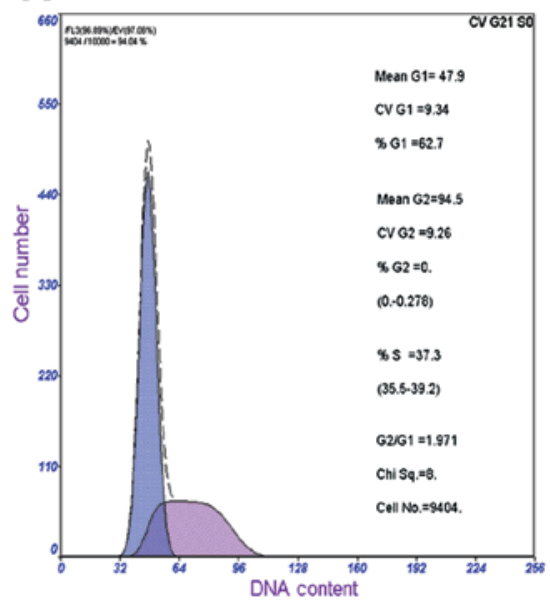

B

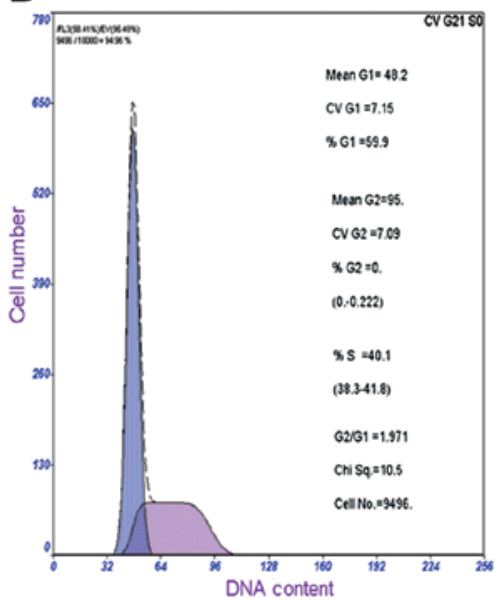

C

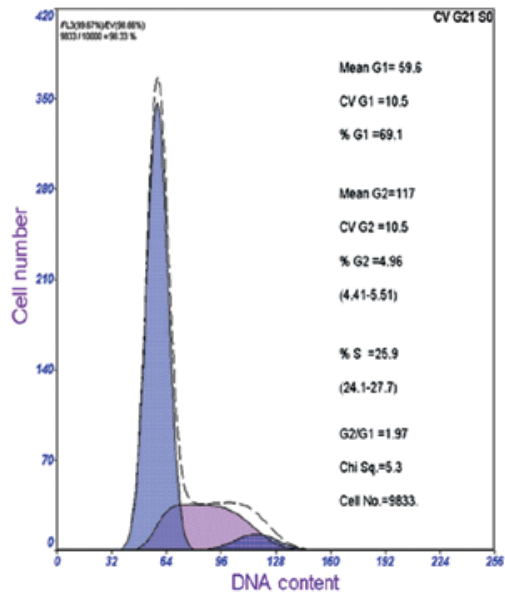

Figure 5. Ornithine decarboxylase antizyme 1 (OAZ1) affects the cell cycle in SCC15 cells. Flow cytometry analysis of (A) untreated SCC15 cells, (B) cells transfected with the control vector and $(\mathrm{C})$ cells transfected with the $O A Z 1$-contained vector.

A

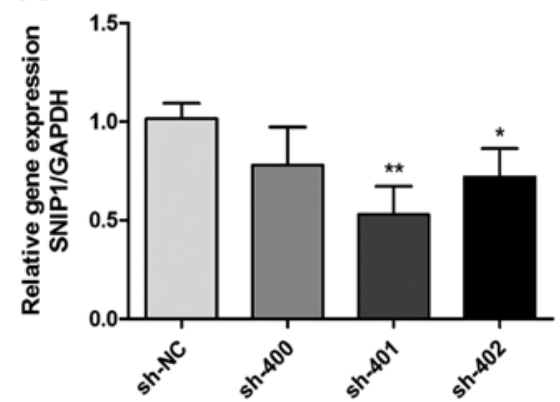

C

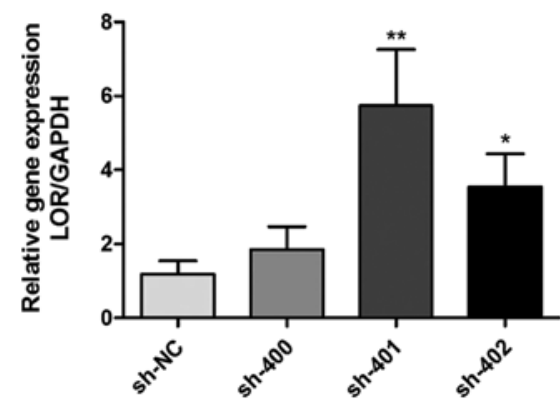

B

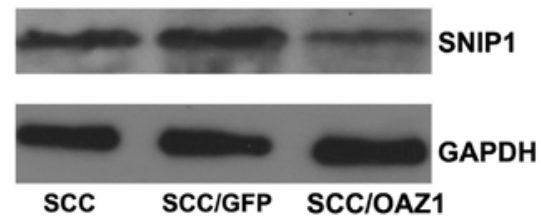

$\operatorname{SCC}$

D

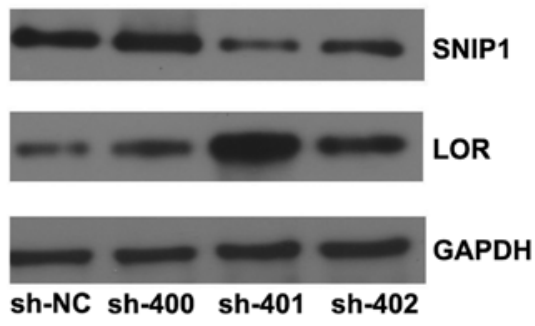

Figure 6. Ornithine decarboxylase antizyme 1 (OAZ1) induced LOR expression through degradation of Smad nuclear interacting protein 1 (SNIP1). Reverse transcription-quantitative polymerase chain reaction (RT-qPCR) analysis of (A) SNIP1 and (C) Loricrin (LOR) expression in the different siRNA silencing groups (sh-400, sh-401 and sh-402) of SNIP1 and the control (sh-NC). (B) Western blot analysis of SNIP1 in the OAZ1-transfected group and control. (D) Western blot analysis of SNIP1 and LOR in the SNIP1-silencing group and the control. ${ }^{*} \mathrm{P}<0.05$ and ${ }^{* *} \mathrm{P}<0.01$ compared to the control group.

(42.47\%) (Table II). These results indicate that OAZ1 may inhibit the cell proliferation through induction of $\mathrm{G}_{0} / \mathrm{G}_{1}$ arrest.

The expression of two genes reported to be elevated during epithelial cell proliferation, $K 6 A$ and $K 19$, were also investigated. RT-qPCR results showed that $K 19$ expression was significantly elevated $(\mathrm{P}<0.05)$, and the level of $K 6 A$ remained stable (Fig. 3B).

OAZ1 inhibits LOR by promoting SNIP1 degradation. OAZ1 can induce the degradation of SNIP1, leading to the release of CREB-binding protein (CBP), a key factor in the regulation of LOR transcription $(14,15)$. To further explore the mechanism of $L O R$ upregulation by OAZ1, the expression of SNIP1 in $O A Z 1$-transfected cells was examined. The SNIP1 protein level was downregulated (Fig. 6B) in $O A Z 1$ transfectants. The RNA interference experiment showed that inhibition of SNIPI in the SCC15 cells induces the expression of $L O R$ (Fig. 6A, C and D). These results indicate that OAZ1 may increase $L O R$ expression through the degradation of SNIP1.

\section{Discussion}

OAZ1 is an important regulator of cellular differentiation and proliferation. Studies have focused on its unique frameshift 
Table II. Effect of OAZ1 on cell cycle in SCC15 cells.

\begin{tabular}{lccc}
\hline Groups & $\mathrm{G}_{1}, \%$ & $\mathrm{~S},(\%)$ & $\mathrm{G}_{2}, \%$ \\
\hline SCC15 & $55.83 \pm 5.95$ & $42.47 \pm 4.49$ & $1.73 \pm 1.57$ \\
SCC15/GFP & $54.83 \pm 4.43$ & $44.17 \pm 3.69$ & $1.36 \pm 1.52$ \\
SCC15/OAZ1 & $65.26 \pm 3.32^{\mathrm{a}}$ & $30.07 \pm 4.42^{\mathrm{b}}$ & $4.64 \pm 2.58$ \\
\hline
\end{tabular}

${ }^{\text {a }}<<0.05$ and ${ }^{\mathrm{b}} \mathrm{P}<0.01$ compared to the other groups. OAZ1, ornithine decarboxylase antizyme 1 .

regulation mechanism, function in regulating polyamine metabolism and ability of inducing ubiquitin-independent degradation of numerous large molecules. Previously, it has been reported that OAZ1 has tumor suppressor activities and it can affect the apoptosis and proliferation of multiple tumor cell lines $(6-8,16)$. There is also evidence that overexpression of $O A Z 1$ can induce the differentiation of certain cancer cells $(10,11,17)$. The mechanism underlying the tumor suppressor activity of OAZ1 remains unclear and further exploration may help with the understanding.

TSCC is one of the most common malignant oral tumors. Previous studies of the oral cancer cell line UM1 have proved that TSCC is an appropriate model to investigate the detailed molecular mechanisms of differentiation and proliferation regulation in oral cancer development $(11,12)$. In the present study, the human TSCC cell line SCC15 was studied and a lentivirial vector was constructed that stably expresses the frame shift form of $O A Z 1$ to continuously trigger the degradation of ODC. The expression of OAZ1 in SCC15 was verified by western blot analysis. Morphological study showed that the cells overexpressing $O A Z 1$ exhibit the formation of more epithelial islands, which is a marker of terminal differentiation of epithelial cells. This is also consistent with the study on the hamster oral cancer cell line, HCPC-1 (10).

To elucidate the mechanism of OAZ1-induced cell differentiation in SCC15 cells, the expression of several terminal differentiation-associated genes was examined, including $K l$, $K 10, F L G, I V L$ and $L O R$. All these genes are marker genes in epithelial differentiation that have decreased expression in epithelial cancer cells, which is resumed during differentiation (18-25). In hamster oral cancer cells, $O A Z 1$ has been proved to promote the expression of $L O R$ and $I V L(9,10)$. In the present study, $O A Z 1$-expressed SCC15 cells showed significantly increased expression of the majority of the marker genes assessed, confirming the positive role of OAZ1 in epithelial differentiation. The gene involved in cellular junction, E-cadherin, was also examined as closer conjunction of cells expressing $O A Z 1$ was observed and E-cadherin is reduced in squamous carcinoma (26). However, the mRNA level of E-cadherin showed no significant difference between the $O A Z 1$-transfected SCC15 and control cells, which is consistent with a previous study (11). This suggests that factors other than E-cadherin may be involved in the induction of closer cellular junctions for this condition.

The mechanism underlying the induction of $L O R$ expression in $O A Z 1$-expressed SCC15 cells was further explored. LOR is the component of the cell membrane in terminal differentiated keratinocytes and p300/CBP can function as a bridge during its transcription-activator complex formation (15). SNIPI has been reported to competitively inhibit the binding of CBP in the promotor region, leading to the inhibition of transcription $(14,27)$. The present study showed that $O A Z 1$ overexpression promoted the degradation of SNIP1 and the silencing of SNIP1 by RNA interference resumed the level of LOR in SCC15 cells. These results indicate that OAZ1 may induce $L O R$ expression partly through SNIP1 degradation.

For the study of cell proliferation in $O A Z 1$-expressing SCC15 cells, the results showed that overexpression of the antizyme significantly inhibited cell proliferation with $\mathrm{G}_{0} / \mathrm{G}_{1}$ arrest. Two genes, $K 6 A$ and $K 19$, were further examined that are elevated during epithelial proliferation $(9,26)$. However, the results of RT-qPCR showed that $K 19$ expression was elevated instead of reduced and the level of K6A remained stable. OAZ1 is assumed to inhibit the proliferation of SCC15 cells without the involvement of regulating K6A and K19 expression.

In conclusion, the study showed that OAZ1 induced the formation of epithelial islands with significant upregulation of epithelial terminal differentiation marker genes in SCC15. OAZ1 expression also inhibited cell proliferation and induced $\mathrm{G}_{0} / \mathrm{G}_{1}$ arrest. OAZ1 simultaneously inhibited proliferation and induced differentiation of oral cancer cells in humans. This may be a novel perspective to consider in the induction of tumor cell differentiation, particularly in human oral cancer.

\section{Acknowledgements}

The present study was supported by the National Natural Science Foundation of China (grant no. 30901757), the Natural Science Foundation of Guangdong Province (grant no. 10151008004000028) and the Leading Talent Project of Guangdong Province.

\section{References}

1. Kahana C: Antizyme and antizyme inhibitor, a regulatory tango. Cell Mol Life Sci 66: 2479-2488, 2009.

2. Newman RM, Mobascher A, Mangold U, et al: Antizyme targets cyclin D1 for degradation. A novel mechanism for cell growth repression. J Biol Chem 279: 41504-41511, 2004.

3. Kasbek C, Yang CH, Yusof AM, Chapman HM, Winey M and Fisk HA: Preventing the degradation of mps1 at centrosomes is sufficient to cause centrosome reduplication in human cells. Mol Biol Cell 18: 4457-4469, 2007.

4. Kasbek C, Yang $\mathrm{CH}$ and Fisk HA: Mps1 as a link between centrosomes and genomic instability. Environ Mol Mutagen 50: 654-665, 2009.

5. Kasbek C, Yang $\mathrm{CH}$ and Fisk HA: Antizyme restrains centrosome amplification by regulating the accumulation of Mps1 at centrosomes. Mol Biol Cell 21: 3878-3889, 2010.

6. Dulloo I, Gopalan G, Melino G, et al: The antiapoptotic DeltaNp73 is degraded in a c-Jun-dependent manner upon genotoxic stress through the antizyme-mediated pathway. Proc Natl Acad Sci USA 107: 4902-4907, 2010.

7. Feith DJ, Origanti S, Shoop PL, Sass-Kuhn S and Shantz LM: Tumor suppressor activity of ODC antizyme in MEK-driven skin tumorigenesis. Carcinogenesis 27: 1090-1098, 2006.

8. Tsuji T, Todd R, Meyer C, et al: Reduction of ornithine decarboxylase antizyme (ODC-Az) level in the 7,12-dimethylbenz(a) anthracene-induced hamster buccal pouch carcinogenesis model. Oncogene 16: 3379-3385, 1998.

9. Feith DJ, Pegg AE and Fong LY: Targeted expression of ornithine decarboxylase antizyme prevents upper aerodigestive tract carcinogenesis in p53-deficient mice. Carcinogenesis 34: 570-576, 2013. 
10. Tsuji T, Usui S, Aida T, et al: Induction of epithelial differentiation and DNA demethylation in hamster malignant oral keratinocyte by ornithine decarboxylase antizyme. Oncogene 20: 24-33, 2001

11. Tsuji T, Katsurano M, Ibaragi S, Shima K, Sasaki A and Hu GF: Ornithine decarboxylase antizyme upregulates DNA-dependent protein kinase and enhances the nonhomologous end-joining repair of DNA double-strand breaks in human oral cancer cells. Biochemistry 46: 8920-8932, 2007.

12. Yamamoto D, Shima K, Matsuo K, et al: Ornithine decarboxylase antizyme induces hypomethylation of genome DNA and histone $\mathrm{H} 3$ lysine 9 dimethylation $(\mathrm{H} 3 \mathrm{~K} 9 \mathrm{me} 2)$ in human oral cancer cell line. PLoS One 5: e12554, 2010.

13. Wu BP, Wang X, Ma WL, Zhang SY, Zheng WL and Jiang L: Effect of ornithine decarboxylase antizyme 1 on the erythroid differentiation in human leukemia cell line K562. Chin J Biochem Mol Biol 29: 361-367, 2013.

14. Lin Y, Martin J, Gruendler C, et al: A novel link between the proteasome pathway and the signal transduction pathway of the bone morphogenetic proteins (BMPs). BMC Cell Biol 3: 15, 2002.

15. Jang SI and Steinert PM: Loricrin expression in cultured human keratinocytes is controlled by a complex interplay between transcription factors of the Sp1, CREB, AP1, and AP2 families. J Biol Chem 277: 42268-42279, 2002.

16. Liu GY, Liao YF, Hsu PC, et al: Antizyme, a natural ornithine decarboxylase inhibitor, induces apoptosis of haematopoietic cells through mitochondrial membrane depolarization and caspases' cascade. Apoptosis 11: 1773-1788, 2006.

17. Suzuki J, Murakami Y, Samejima K, Ohtani KK and Oka T: Antizyme is necessary for conversion of pancreatic tumor cells into glucagon-producing differentiated cells. Endocr Relat Cancer 16: 649-659, 2009.

18. Wu N, Sulpice E, Obeid P, Benzina S, Kermarrec F, Combe S and Gidrol X: The miR-17 family links p63 protein to MAPK signaling to promote the onset of human keratinocyte differentiation. PLoS One 7: e45761, 2012.
19. Woelfle U, Laszczyk M N, Kraus M, et al: Triterpenes promote keratinocyte differentiation in vitro, ex vivo and in vivo: a role for the transient receptor potential canonical (subtype) $6 . J$ Invest Dermatol 130: 113-123, 2010

20. Robertson ED, Weir L, Romanowska M, Leigh IM and Panteleyev AA: ARNT controls the expression of epidermal differentiation genes through HDAC- and EGFR-dependent pathways. J Cell Sci 125: 3320-3332, 2012.

21. Chan K S, Sano S, Kataoka K, et al: Forced expression of a constitutively active form of Stat 3 in mouse epidermis enhances malignant progression of skin tumors induced by two-stage carcinogenesis. Oncogene 27: 1087-1094, 2008.

22. Kawachi Y, Fujisawa Y, Furuta J, Nakamura Y, Ishii Y and Otsuka F: Superficial epithelioma with sebaceous differentiation: immunohistochemical study of keratinocyte differentiation markers. Eur J Dermatol 21: 1016-1017, 2011.

23. Tu CL, Chang W and Bikle DD: The calcium-sensing receptor-dependent regulation of cell-cell adhesion and keratinocyte differentiation requires Rho and filamin A. J Invest Dermatol 131: 1119-1128, 2011

24. Cohen I, Birnbaum R Y, Leibson K, Taube R, Sivan S and Birk OS: ZNF750 is expressed in differentiated keratinocytes and regulates epidermal late differentiation genes. PLoS One 7: e42628, 2012.

25. Blander G, Bhimavarapu A, Mammone T, et al: SIRT1 promotes differentiation of normal human keratinocytes. J Invest Dermatol 129: 41-49, 2009.

26. Gasparoni A, Fonzi L, Schneider GB, Wertz PW, Johnson GK and Squier CA: Comparison of differentiation markers between normal and two squamous cell carcinoma cell lines in culture. Arch Oral Biol 49: 653-664, 2004.

27. Kim RH, Flanders KC, Birkey Reffey S, Anderson LA, Duckett CS, Perkins ND and Roberts AB: SNIP1 inhibits NF-kappa B signaling by competing for its binding to the $\mathrm{C} / \mathrm{H} 1$ domain of $\mathrm{CBP} / \mathrm{p} 300$ transcriptional co-activators. J Biol Chem 276: 46297-46304, 2001. 